OPEN ACCESS

Edited by:

Wafik S. El-Deiry,

Brown University, United States

Reviewed by:

Wen-i Chang,

Lifespan, United States

Zhifang Zhang,

City of Hope, United States

*Correspondence:

Fei Peng

pengf_whu@sina.com

Specialty section:

This article was submitted to Cancer Molecular Targets and Therapeutics,

a section of the journa

Frontiers in Oncology

Received: 09 June 2020 Accepted: 14 December 2020

Published: 05 February 2021

Citation:

Peng F, Cai W, Li J and Li H (2021) ClC-5 Downregulation Induces

Osteosarcoma Cell Apoptosis

by Promoting Bax and tBid

Complex Formation.

Front. Oncol. 10:556908.

doi: 10.3389/fonc.2020.556908

\section{CIC-5 Downregulation Induces Osteosarcoma Cell Apoptosis by Promoting Bax and tBid Complex Formation}

\author{
Fei Peng ${ }^{*}$, Weisong Cai, Jianping Li and Haohuan Li \\ Department of Orthopedics, Renmin Hospital of Wuhan University, Wuhan, China
}

Osteosarcoma is the most common malignant bone tumor. Chloride $\left(\mathrm{Cl}^{-}\right)$channelsmediated $\mathrm{Cl}^{-}$movement plays an important role in regulating the functions of various cancer cells, but its role in osteosarcoma remains unclear. In this study, we found that ClC-5 was increased in osteosarcoma tissues compared with normal bone tissues. Patients with high $\mathrm{ClC}-5$ expression showed poor overall survival relative to those patients with low ClC-5 expression. Higher ClC-5 expression and lower intracellular $\mathrm{Cl}^{-}$ concentration $\left(\left[\mathrm{Cl}^{-}\right]_{\mathrm{i}}\right)$ were observed in osteosarcoma cells compared with normal osteoblasts. Lowering $\left[\mathrm{Cl}^{-}\right]_{\mathrm{i}}$ increased the viability of osteosarcoma cells, which was markedly blocked by $\mathrm{ClC}-5$ downregulation. Knockdown of $\mathrm{CIC}-5$ significantly induced osteosarcoma cell apoptosis and increased the release of cytochrome c from mitochondria to cytosol, concomitantly with cleavage of caspase-9, caspase-3, and PARP. The effect of CIC-5 downregulation on osteosarcoma cell apoptosis and viability was abolished by caspase- 3 and caspase- 9 inhibitors, but not caspase- 8 inhibitor. Furthermore, ClC-5 inhibition promoted Bax translocation from cytosol to mitochondria. Immunoprecipitation showed that $\mathrm{ClC}-5$ interacted with $\mathrm{Bax}$ and $\mathrm{ClC}-5$ downregulation enhanced Bax and tBid complex formation. Collectively, we demonstrate that $\mathrm{ClC}-5$ downregulation induces osteosarcoma cell apoptosis via mitochondria-dependent apoptotic pathway activation by promoting Bax and tBid association and subsequent Bax translocation.

Keywords: osteosarcoma, chloride, CIC-5, apoptosis, mitochondria, Bax

\section{INTRODUCTION}

Osteosarcoma is the most common primary malignant bone tumor in children and adolescents (1). It accounts for about $20 \%$ of primary bone cancer and $2.4 \%$ of all malignancies in pediatric patients (2). Osteosarcoma patients usually receive a three-drug chemotherapy regimen consisting of cisplatin, doxorubicin, and methotrexate, followed by surgical resection of the primary tumor in which higher survival rates have been achieved $(3,4)$. Unfortunately, some patients are diagnosed with advanced cancer at the first diagnosis. The therapeutic effects of surgery in this stage are poor due to the distant metastasis (1). Thus, it may be of great importance to understand the molecular 
mechanisms of osteosarcoma development and identify a novel biomarker that could be used for osteosarcoma diagnosis.

Chloride $\left(\mathrm{Cl}^{-}\right)$, the most common and abundant anions in organism, plays a key role in regulating various cellular and intracellular functions (5). $\mathrm{Cl}^{-}$movement resulting in decrease of intracellular $\mathrm{Cl}^{-}$concentration $\left(\left[\mathrm{Cl}^{-}\right]_{\mathrm{i}}\right)$ is closely related to diverse physiological processes, including vascular inflammation, foam cell formation, hypertension, and cancer drug resistance (6-8). It has been known that the $\mathrm{Cl}^{-}$movement is strictly regulated by $\mathrm{Cl}^{-}$channels, a kind of permeable channels or proteins encoded by the genes of $\mathrm{ClC}$ family. $\mathrm{ClC}-5$, a member of $\mathrm{ClC}$ family, is recently suggested to be associated with the development of several kinds of cancers $(9,10)$. It has been reported that $\mathrm{ClC}-5$ is highly expressed in glioma cells and leukemic cells $(11,12)$. Moreover, ClC-5 overexpression decreased drug sensitivity in multiple myeloma cells via promoting pro-survival autophagy (9). Nevertheless, the functional role of $\mathrm{Cl}^{-}$movement and ClC-5 in osteosarcoma remains unknown. In this study, we investigated the correlation between $\mathrm{ClC}-5$ and the growth of osteosarcoma cells, demonstrating that the increased $\mathrm{ClC}-5$ expression may facilitate osteosarcoma cell growth and predict poor prognosis of osteosarcoma. Our findings suggest that targeting $\mathrm{ClC}-5$ may be a potential strategy for the treatment of osteosarcoma.

\section{METHODS AND MATERIALS}

\section{Materials and Reagents}

RPMI1640 medium, fetal bovine serum (FBS), penicillin, and streptomycin were obtained from Invitrogen (CA, USA). 6methoxy-N-ethyl-1,2-dihydroquinoline (dihydro-MEQ), and antibodies against $\mathrm{ClC}-5$ and GAPDH were obtained from Sigma (MO, USA). Primary antibodies against cytochrome c, cleaved caspase-9, cleaved caspase-3, cleaved caspase-8, cleaved PARP, and horse peroxidase (HRP) conjugated secondary antibodies were purchased from Cell Signaling Technology (MA, USA). Bax, tBid, and COX IV antibodies were obtained from Santa Cruz Biotechnology (CA, USA). Specific caspase-9 inhibitor Z-LEHD-FMK, specific caspase-8 inhibitor Z-IETDFMK, and specific caspase-3 inhibitor Z-VAD-FMK were purchased from Calbiochem (Darmstadt, Germany).

\section{Control and Patient Osteosarcoma Samples}

This study included 30 osteosarcoma patients who received surgical biopsy with no prior chemotherapy or radiation therapy between 2007 and 2008 in the Renmin Hospital of Wuhan University in Wuhan, China. Meanwhile, 30 cases of normal bone tissues were considered as control. The normal bone tissues were resected within at least $5 \mathrm{~cm}$ to the margin of tumor. After surgical resection, all the osteosarcoma and normal bone tissues were frozen within $30 \mathrm{~min}$.

\section{Immunohistochemistry}

Immunohistochemistry for ClC-5 was performed on $4-\mu \mathrm{m}$ paraffin-embedded sections from human osteosarcoma tissues using the streptavidin-biotin-peroxidase complex system according to the supplier's instructions (DAKO Japan, Tokyo, Japan). The sections were heated for $30 \mathrm{~min}$ at $65^{\circ} \mathrm{C}$, dewaxed in xylene, and rehydrated by $100,95,70$, and $50 \%$ alcohols at room temperature for $1 \mathrm{~min}$. The sections were blocked by $10 \%$ goat serum and incubated with $\mathrm{ClC}-5$ antibody overnight at $4^{\circ} \mathrm{C}$, followed by incubation with biotinylated secondary antibody. The expression of ClC-5 was visualized with the streptavidinperoxidase reaction using 3,3'-diaminobenzidine under CKX41 optical microscope (Olympus, Japan).

\section{Cell Culture and Transfection}

The human normal osteoblasts hFOB1.19, osteosarcoma cell lines U-2OS, SaoS-2 and HOS, and human renal proximal tubule epithelial HK-2 cells were purchased from China Center Type Culture Collection (CCTCC, Shanghai, China). Cells were cultured in RPMI1640 supplemented with $10 \%$ FBS, $100 \mathrm{U} / \mathrm{ml}$ penicillin and $100 \mu \mathrm{g} / \mathrm{ml}$ streptomycin in a humidified atmosphere of $5 \% \mathrm{CO} 2$ at $37^{\circ} \mathrm{C}$. The sequence of the stealth siRNA duplex oligoribonucleotides against human $\mathrm{ClC}-5$ gene (5'-GCACTTCCATCATTCATTT-3') and negative siRNA were synthesized and purchased from Santa Cruz Biotechnology. The siRNAs were transfected transiently with Lipo RNAi max Reagent (Applied Biosystems, CA, USA) for $48 \mathrm{~h}$ according to the manufacturer's instructions.

\section{Low $\mathrm{Cl}^{-}$Medium Preparation}

Low $\mathrm{Cl}^{-}$medium was prepared as previously described (8). Briefly, RPMI1640 medium lacking $\mathrm{NaCl}$ and $\mathrm{KCl}$ was also obtained from Invitrogen. The $5 \mathrm{mmol} / \mathrm{L} \mathrm{KCl}$ and $105 \mathrm{mmol} / \mathrm{L}$ $\mathrm{NaCl}$ in normal $\mathrm{Cl}^{-}$medium were replaced by $5 \mathrm{mmol} / \mathrm{L}$ potassium gluconate and $105 \mathrm{mmol} / \mathrm{L}$ sodium gluconate $(\mathrm{pH}=$ 7.2). The osmolarities of the solutions were measured by a freezing point depression osmometer (OSMOMAT030, Gonotec GmbH, Berlin, Germany).

\section{Intracellular $\mathrm{Cl}^{-}$Concentration $\left(\left[\mathrm{Cl}^{-}\right]_{\mathrm{i}}\right)$ Measurement}

$\left[\mathrm{Cl}^{-}\right]_{\mathrm{i}}$ was measured as previously described $(6,8)$. Osteosarcoma cells were incubated with dihydro-MEQ $(100 \mu \mathrm{mol} / \mathrm{L})$ in a Ringer's buffer solution containing (in mmol/L: $119 \mathrm{NaCl}, 2.5$ $\mathrm{KCl}, 1.0 \mathrm{NaH}_{2} \mathrm{PO}_{4}, 1.3 \mathrm{MgSO}_{4}, 2.5 \mathrm{CaCl}_{2}, 26 \mathrm{NaHCO}_{3}, 11$ glucose, $\mathrm{pH}$ 7.4) at room temperature in the dark for $30 \mathrm{~min}$. Then dihydro-MEQ was oxidized to MEQ. The fluorescence of MEQ was monitored by MetaFluor Imaging software (Universal Imaging Systems, Chester, PA, USA) with 350-nm excitation and 435-nm emission wavelength. $\left[\mathrm{Cl}^{-}\right]_{\mathrm{i}}$ was calculated by the SternVolmer equation: $(\mathrm{FO} / \mathrm{F})-1=\mathrm{K} \mathrm{SV}[\mathrm{Q}]$. FO is the fluorescence intensity without quencher; $\mathrm{F}$ is the fluorescence intensity in the presence of quencher; [Q] is the concentration of quencher; and $\mathrm{KSV}$ is the Stern-Volmer constant.

\section{Cell Viability Assay}

Cell viability was determined by cell counting kit-8 (CCK-8; Yiyuan Biotechnology, Guangzhou, China) according to the manufacturer's protocols. After corresponding treatment, $10 \mu \mathrm{l}$ of CCK8 was added to each well for additional $3 \mathrm{~h}$ at $37^{\circ} \mathrm{C}$. The 
absorbance of each well was measured with a SPECTRA MAX190 spectrophotometry (Sunnyvale, CA, USA) at 450 nm wavelength.

\section{Real-Time PCR}

The osteosarcoma samples were homogenized in TRIzol reagent (Invitrogen, CA, USA) and total RNA was isolated according to the manufacturer's instructions. One microgram of RNA was reverse-transcribed to cDNA according to the manufacturer's instructions (Thermo Fisher Scientific Inc., IL, USA). A SYBR QPCR Kit (Toyobo, Osaka, Japan) was used in associated with ABI 7500 real-time PCR system (Applied Biosystems, CA, USA) to detect ClC-5 mRNA expression. Human $18 \mathrm{~S}$ rRNA was used as an endogenous control. The primer sequences were as follows: ClC-5, 5'-GTGAGGGAGAAATCCAGA-3' and 5'-TTGAT GATCAGCGTCCA-3'; $18 \mathrm{~S}$ rRNA, 5'-CGGCTAC CACATCCAAGGAA-3' and 5'-CTGGAATTACCGCGGCT-3'.

\section{Western Blot Analysis and Immunoprecipitation}

Osteosarcoma cells were harvested and lysed in RIPA lysis buffer containing protease and phosphatase inhibitor cocktail (Beyotime, Jiangsu, China) and the protein was isolated according to the manufacturer's instructions. The protein content was quantified using a micro BCA kit (Beyotime). The samples containing equal protein $(100 \mu \mathrm{g})$ were diluted in loading buffer and heated for $10 \mathrm{~min}$ at $99^{\circ} \mathrm{C}$, before separated on $8-12 \%$ SDS-PAGE. Separated proteins were transferred onto polyvinylidene fluoride membranes (Millipore, MA, USA) at 200 $\mathrm{mA}$ for $90 \mathrm{~min}$. Unspecific banding sites on the membrane were blocked by $5 \%$ non-fat milk. The following antibodies were used: ClC-5 (1:500), cytochrome c, cleaved caspase-9, cleaved caspase3, cleaved caspase-8, cleaved PARP, Bax, tBid, COX IV, and GAPDH (1:1,000). After washing, membranes were incubated with appropriate HRP conjugated secondary antibody $(1: 1,000)$. Bands were detected and visualized by Amersham BiosciencesTM ECLTM Western blotting detection reagent. Densitometric analysis was conducted with Image J software (National Institutes of Health, MD, USA). For immunoprecipitation, equal cellular proteins were incubated with limiting amounts of Bax antibody overnight at $4^{\circ} \mathrm{C}$ with constant rotation. The complexes were collected following incubation with protein $\mathrm{A} / \mathrm{G}$ agarose beads for $4 \mathrm{~h}$ at $4^{\circ} \mathrm{C}$ and resuspended in RIPA lysis buffer for western blot analysis using tBid antibody.

\section{Immunofluorescence Staining}

To detect ClC-5 localization, osteoblast and osteosarcoma cells were fixed and labeled with ClC-5 antibody (1:100) overnight at $4^{\circ} \mathrm{C}$, followed by incubation with TRITC-labeled secondary antibody (1:200, Jackson Laboratory, ME, USA) for $1 \mathrm{~h}$ at room temperature. Fluorescent images were acquired using the Zeiss LSM 710 laser-scanning confocal microscopy (Munich, Germany).

\section{Flow Cytometry}

The apoptosis of osteosarcoma cells was detected with the FITCAnnexin V Apoptosis Detection Kit (Nanjing Jiancheng, Jiangsu,
China) using a flow cytometry (BD Biosciences, Palo Alto, CA, USA). In brief, cells were trypsinized and harvested by centrifugation. The cell pallets were washed with PBS, resuspended, and incubated with Annexin $\mathrm{V}$ and propidium iodide (PI) at room temperature in dark for $20 \mathrm{~min}$. The stained cells were analyzed by a flow cytometry and data were analyzed by CFlow Plus software (BD Biosciences).

\section{TUNEL Staining}

Cell apoptosis was also detected by TUNEL assay using an In situ Cell Death Detection kit (Roche, Mannheim, Germany). The cells were fixed with $4 \%$ paraformaldehyde for $30 \mathrm{~min}$ at room temperature, permeabilized with $0.1 \%$ Triton X-100 for $5 \mathrm{~min}$, and incubated with TUNEL Reaction Mixture for $60 \mathrm{~min}$ at $37^{\circ} \mathrm{C}$. The nuclei were counterstained with DAPI. Apoptotic cells were viewed using the Zeiss LSM 710 laser-scanning confocal microscopy. The number of TUNEL positive cells (red) were counted using Image J software. The percentage of TUNELpositive cells was expressed as TUNEL positive cells/field.

\section{Mitochondrial Fractions Preparation}

Mitochondrial and cytoplasmic proteins were isolated using the Mitochondria Isolation Kit (Thermo Fisher Scientific Inc., IL, USA) according to the manufacturer's protocol. These fractions were analyzed by western blot analysis. COX IV was used as a loading control for the mitochondria fraction.

\section{Statistical Analyses}

Continuous variables are expressed as mean \pm SEM. The ANOVA (post hoc test: Tukey) was used to analyze the differences between groups for experiments with more than two subgroups. Survival curves were estimated using Kaplan-Meier method and compared by log rank test. Data were statistically analyzed by SPSS15.0 statistical software (SPSS Inc., IL, USA). $\mathrm{P}$ value $<0.05$ were considered statistically significant.

\section{RESULTS}

\section{Increased CIC-5 Expression in Osteosarcoma Tissues}

To investigate the role of $\mathrm{ClC}-5$ in osteosarcoma, we first utilized immunohistochemistry to study the expression files of ClC-5 in normal bone tissues and osteosarcoma tissues. As shown in Figure 1A, in human osteosarcoma tissues, ClC-5 was significantly overexpressed compared with normal bone tissues. Similar to this result, both real-time PCR and western blotting revealed that the expression of $\mathrm{ClC}-5$ was increased in osteosarcoma tissues compared with normal bone tissues (Figures 1B, C). To further determine the relationship between ClC-5 expression and the clinic pathologic characteristics of osteosarcoma patients, the patients were divided into low expression group and high expression group according to the median expression of $\mathrm{ClC}-5$. We found that the ClC-5 expression was in association with tumor size, grade, distant metastasis, and clinical stage. However, the expression 
A

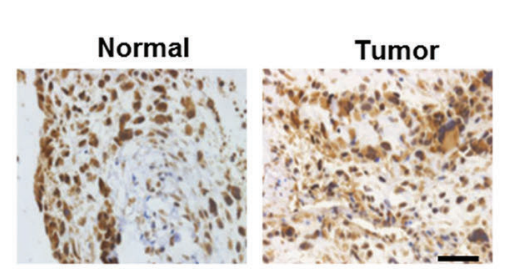

D

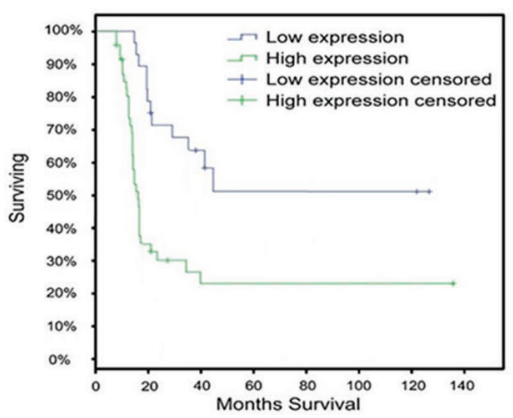

G

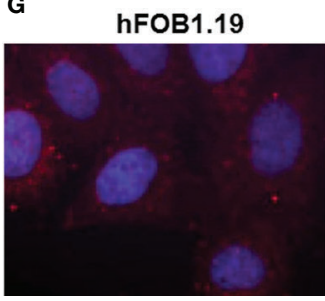

B

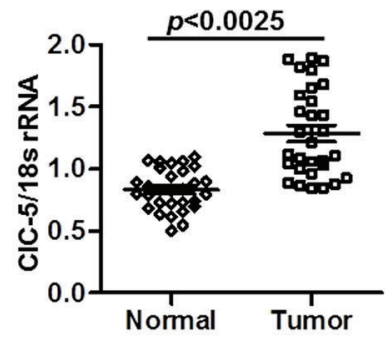

E

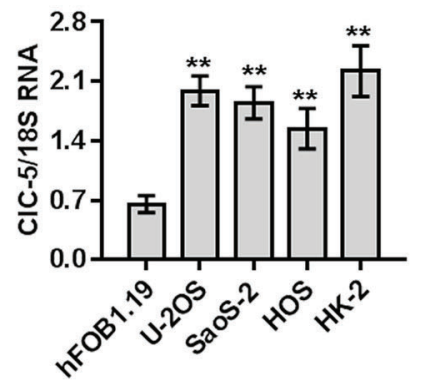

Saos-2
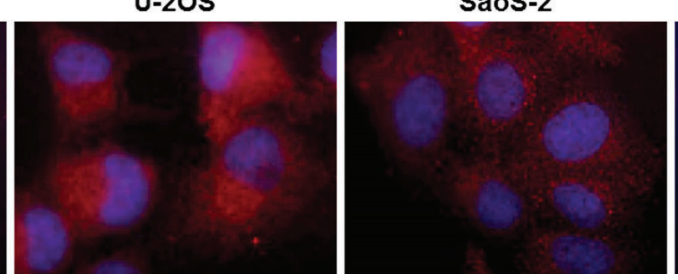

C
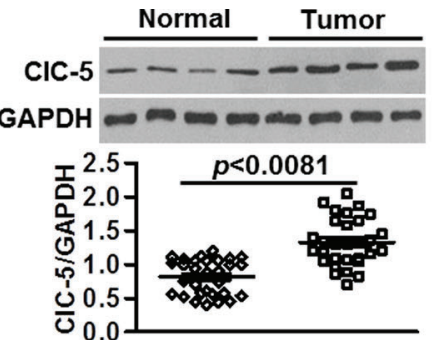

F

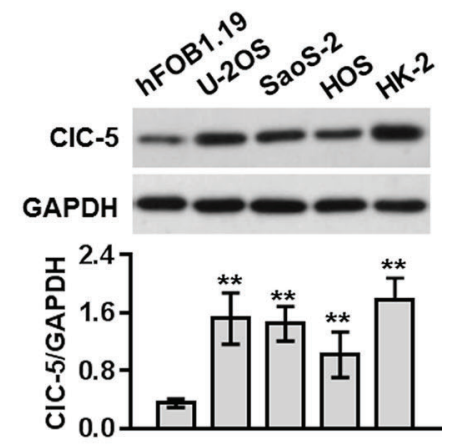

HOS

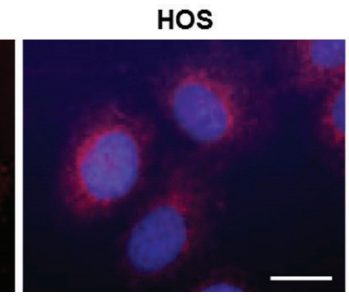

FIGURE 1 | ClC-5 expression is increased in osteosarcoma tissues. (A) Paraffin-embedded sections of normal bone tissues and osteosarcoma tissues were collected. Expression of CIC-5 was detected by immunohistochemistry. Scale bar, $100 \mu \mathrm{m}$. (B) Real-time PCR analysis to quantify the endogenous expression of CIC-5 in normal bone tissues $(n=30)$ compared with osteosarcoma tissues $(n=30)$. (C) ClC-5 protein expression was determined by Western blotting and quantitative results of ClC-5 expression were presented. (D) Correlation of CIC-5 expression and overall survival in patients with osteosarcoma cancer. Observations were censored at trial discontinuation for reasons other than death or at trial completion. (E, F) The CIC-5 expression in human normal osteoblasts hFOB1.19, osteosarcoma cell lines U-2OS, SaoS-2, and HOS, and renal proximal tubule epithelial HK-2 cells was determined by real-time PCR (E) and western blotting (F). ${ }^{* *} \mathrm{P}<0.01$ vs. hFOB1.19 cells, $\mathrm{n}=6$. (G) Representative images of $\mathrm{CIC}-5$ distributions in osteoblast and osteosarcoma cells. $\mathrm{N}=4$. Scale bar, $20 \mu \mathrm{m}$.

of ClC-5 in osteosarcoma patients had no correlation with age and gender (Table 1). Moreover, Kaplan-Meier survival analysis showed that ClC-5-high patients were significantly connected with poor prognosis, indicating that the osteosarcoma patients with high ClC-5 expression have lower overall survival than those with $\mathrm{ClC}$-5-low expression ( $\mathrm{P}=0.02$ log-rank test) (Figure 1D). In vitro, the abundance of expression of $\mathrm{ClC}-5$ was significantly higher in osteosarcoma cell lines compared with normal osteoblasts hFOB1.19, and even close to that in renal proximal tubule epithelial HK-2 cells (Figures 1E, F), where ClC-5 has been demonstrated to be highly expressed (13). The subcellular localization of ClC-5 in osteoblast and osteosarcoma cells has not been previously explored. Immunofluorescence staining revealed that $\mathrm{ClC}-5$ accumulated around the nucleus, and exhibited a punctate distribution, which extended throughout the cell (Figure 1G). Collectively, these results suggest that increased expression of ClC-5 may be associated with the development of osteosarcoma.

\section{CIC-5-Mediated $\mathrm{Cl}^{-}$Efflux Promotes the Growth of Osteosarcoma Cells}

As ClC-5 is an important $\mathrm{Cl}^{-}$channel, we thus examined whether $\left[\mathrm{Cl}^{-}\right]_{\mathrm{i}}$ is changed in osteosarcoma cells. The results showed that $\left[\mathrm{Cl}^{-}\right]_{\mathrm{i}}$ was markedly decreased in osteosarcoma cells compared with normal osteoblasts (Figure 2A). To explore the physiological function of the reduced $\left[\mathrm{Cl}^{-}\right]_{\mathrm{i}}$, low $\mathrm{Cl}^{-}$medium was prepared and cell viability was assessed. CCK- 8 assay revealed that lowering $\left[\mathrm{Cl}^{-}\right]_{\mathrm{i}}$ increased the viability of $\mathrm{U}-2 \mathrm{OS}$, SaoS-2, and HOS cells, but had no effect on control osteoblasts cell line hFOB1.19 (Figure 2B). We next investigated that whether the increased $\mathrm{ClC}-5$ expression is responsible for the lowering $\left[\mathrm{Cl}^{-}\right]_{\mathrm{i}}$-induced osteosarcoma cell growth, ClC-5 
TABLE 1 | Relationship between ClC-5 expression and the clinical characteristics of the osteosarcoma patients.

\begin{tabular}{|c|c|c|c|}
\hline & \multicolumn{2}{|c|}{ CIC-5 expression } & \multirow[t]{2}{*}{$P$ values } \\
\hline & Low $(n=14)$ & High $(n=16)$ & \\
\hline Age (years) & & & 0.8731 \\
\hline$\geq 18$ & 10 & 11 & \\
\hline$<18$ & 4 & 5 & \\
\hline Gender & & & 0.3006 \\
\hline Female & 8 & 12 & \\
\hline Male & 6 & 4 & \\
\hline Tumor size (cm) & & & 0.0111 \\
\hline$<10$ & 9 & 3 & \\
\hline$\geq 10$ & 5 & 13 & \\
\hline Grade & & & 0.0024 \\
\hline Low & 8 & 1 & \\
\hline High & 6 & 15 & \\
\hline Distant metastasis & & & 0.0235 \\
\hline Negative & 11 & 6 & \\
\hline Positive & 3 & 10 & \\
\hline Stage & & & 0.0480 \\
\hline 1 & 8 & 3 & \\
\hline$\|$ & 5 & 7 & \\
\hline III & 1 & 6 & \\
\hline
\end{tabular}

expression was inhibited by siRNA under normal and low $\mathrm{Cl}^{-}$ conditions (Figure S1). Inhibition of $\mathrm{ClC}-5$ not only reduced osteosarcoma cell viability in normal $\mathrm{Cl}^{-}$medium, but also attenuated lowering $\left[\mathrm{Cl}^{-}\right]_{\mathrm{i}}$-induced the increase in cell viability (Figures 2C-E). However, ClC-5 downregulation exerted no effect on hFOB1.19 cell viability when the cells were cultured with or without low $\mathrm{Cl}^{-}$medium (Figure 2F).

\section{Downregulation of CIC-5 Induces Osteosarcoma Cell Apoptosis}

To test whether the decreased cell viability by ClC-5 downregulation results from osteosarcoma cell apoptosis, cell apoptosis was analyzed by Annexin V-FITC/PI flow cytometry and TUNEL staining. The Annexin V-FITC/PI data showed that ClC-5 knockdown increased the apoptotic rate to $32.4 \pm 5.5 \%$ and $27.8 \pm 3.6 \%$ in U-2OS and SaoS-2 cells, respectively (Figures 3A, B). Moreover, as displayed in Figure 3C, there were little TUNELpositive cells in control groups. However, the apoptotic cells were markedly increased by ClC- 5 downregulation. The statistical result of the percentage of TUNEL-positive cells validated the alteration in osteosarcoma cell apoptosis (Figure 3D).

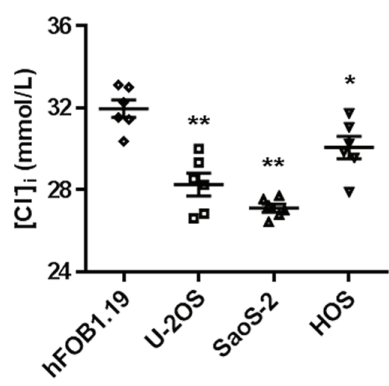

D

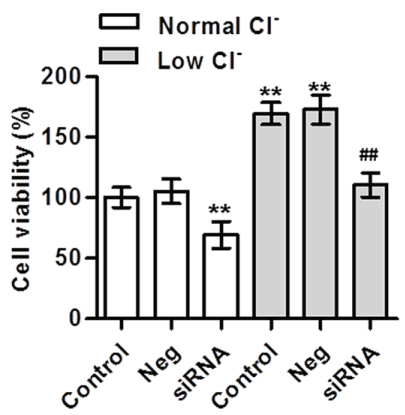

B

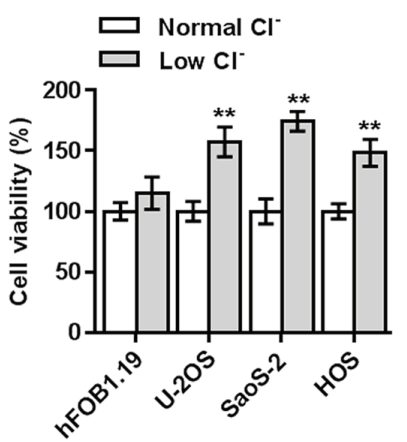

E

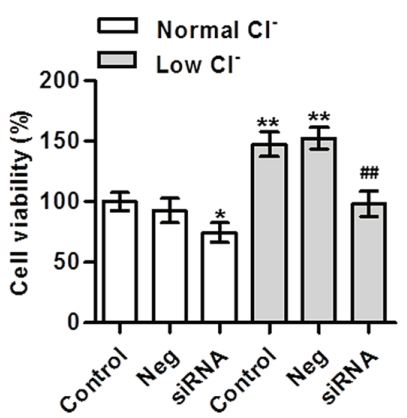

C

$\mathrm{U}-20 \mathrm{~S}$
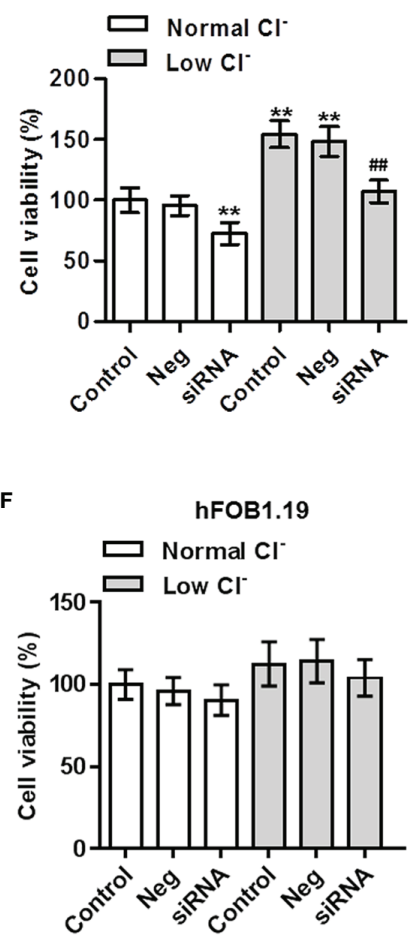

FIGURE 2 | Inhibition of CIC-5 attenuates low $\mathrm{Cl}^{-}$-induced the increase in osteosarcoma cell viability. (A) The intracellular chloride concentration ([Cl- $]$) in $\mathrm{hFOB1.19,}$ U-2OS, SaoS-2, and HOS cells was examined using MEQ fluorescence probe. ${ }^{\star} \mathrm{P}<0.05$, ${ }^{\star \star} \mathrm{P}<0.01$ vs. $\mathrm{hFOB} 1.19$ cells, $\mathrm{n}=6$. (B) $\mathrm{hFOB1} .19, \mathrm{U}-2 \mathrm{OS}, \mathrm{SaoS}-2$, and HOS cells were incubated in normal medium or low $\mathrm{Cl}^{-}$medium for $24 \mathrm{~h}$. Cell viability was assessed by CCK-8 assay. ${ }^{* *} \mathrm{P}<0.01 \mathrm{vs} . \mathrm{normal} \mathrm{Cl}^{-}$, respectively, $\mathrm{n}=6$. (C-F) U-2OS (C), SaoS-2 (D), HOS (E), and hFOB1.19 (F) cells were transfected with ClC-5 siRNA or negative siRNA (Neg) for $48 \mathrm{~h}$ and then treated with low Cl${ }^{-}$ medium for $24 \mathrm{~h}$. Cell viability was determined. ${ }^{\star \star} \mathrm{P}<0.01 \mathrm{vs}$. normal $\mathrm{Cl}^{-}+$control; ${ }^{\# \#} \mathrm{P}<0.01$ vs. low $\mathrm{Cl}^{-}+$control, $\mathrm{n}=6$. 
A

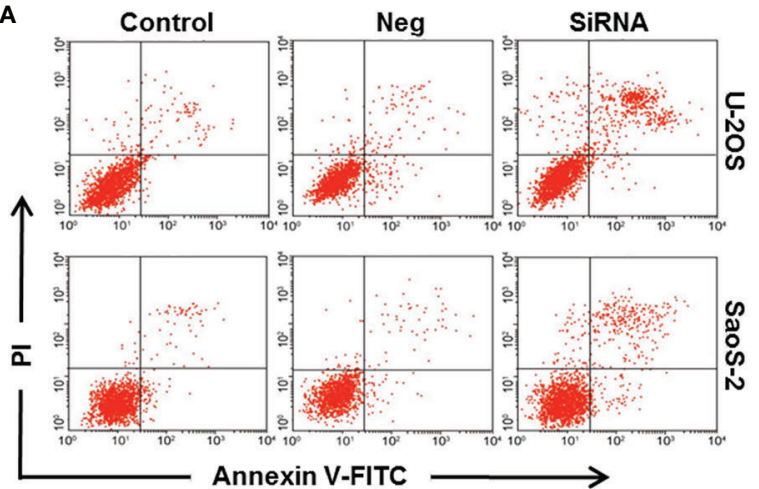

C

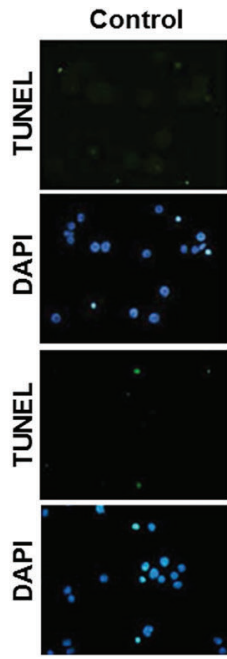

Neg

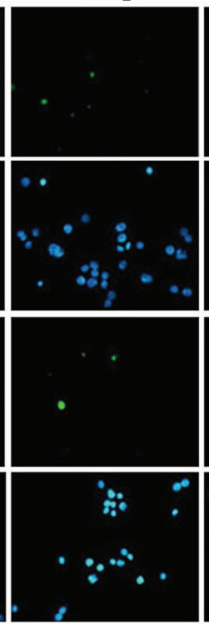

SiRNA

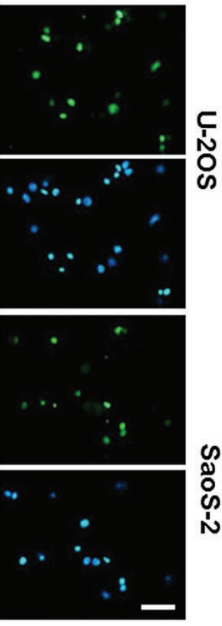

B

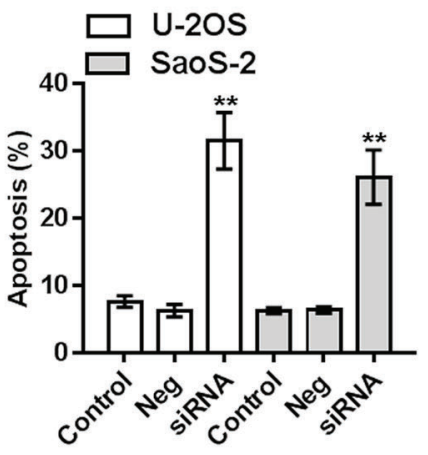

FIGURE 3 | CIC-5 downregulation induces apoptosis in osteosarcoma cells. (A) U-2OS and SaoS-2 cells were transfected with CIC-5 siRNA or negative siRNA (Neg) for $48 \mathrm{~h}$. Cell apoptosis was determined by Annexin V/PI staining. (B) Quantitative analysis of the percentage of apoptotic cells. (C) Apoptosis was also detected by TUNEL staining. Representative images were shown. (D) Quantification of apoptotic osteosarcoma cells. Scale bar, $20 \mu \mathrm{m}$. ${ }^{\star \star} \mathrm{P}<0.01$ vs. corresponding control, $\mathrm{n}=6$.

\section{CIC-5 Knockdown Enhances \\ Osteosarcoma Cell Apoptosis via Mitochondria-Dependent Apoptotic Pathway}

There are two major apoptotic pathways, namely extrinsic and intrinsic (mitochondria-dependent) pathways $(14,15)$. To examine the mechanism how $\mathrm{ClC}-5$ downregulation causes osteosarcoma cell apoptosis, we initially tested the expression of cytochrome $\mathrm{c}$ in the soluble cytosolic fraction. Western blotting showed that ClC-5 knockdown significantly induced cytochrome c release from mitochondria to cytosol in U-2OS and SaoS- 2 cells. Furthermore, the cleavage of caspase-9/-3 and subsequent cleavage of PARP were also increased in cells transfected with ClC-5 siRNA. However, ClC-5 downregulation had no effect on the extrinsic pathway initiator caspase- 8 cleavage, indicating extrinsic pathway may be not involved (Figures 4 A-C). We next treated ClC-5 siRNA-transfected osteosarcoma cells with different caspase inhibitors and measured their effect on cell apoptosis and viability. Inhibitors experiments showed that ClC-5 downregulation-induced the increase in cell apoptosis was significantly inhibited by caspase-9 inhibitor Z-LEHD-FMK or caspase-3 inhibitor Z-VAD-FMK, but not caspase-8 inhibitor Z-IETD-FMK produced no effects (Figures 4D, E). Consistently, CCK-8 assay revealed that caspase-9 inhibitor and caspase-3 inhibitor but not caspase- 8 inhibitor increased the viability of U-2OS and SaoS-2 cells that were transfected with ClC-5 siRNA (Figures 4F, G). These results indicate that intrinsic mitochondria-dependent pathway may be the major mechanism responsible for the apoptotic effects of ClC-5 downregulation in osteosarcoma cells.

\section{CIC-5 Interacts Bax and Induces Its Translocation}

The release of cytochrome $\mathrm{c}$ is usually triggers by the translocation of Bax from cytosol to mitochondria (15). As shown in Figures 5A, B, ClC-5 inhibition markedly decreased 


\section{A}

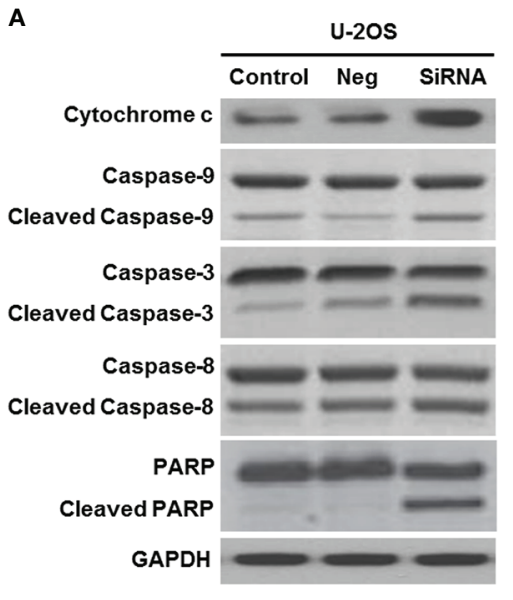

B

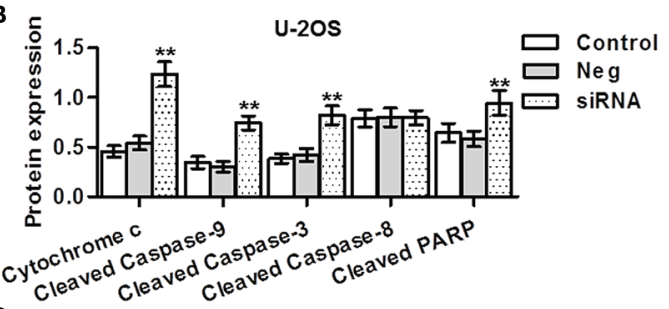

C

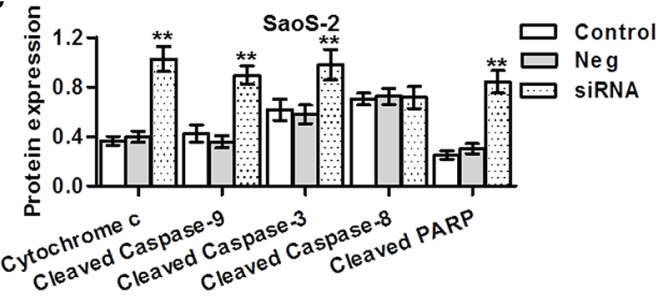

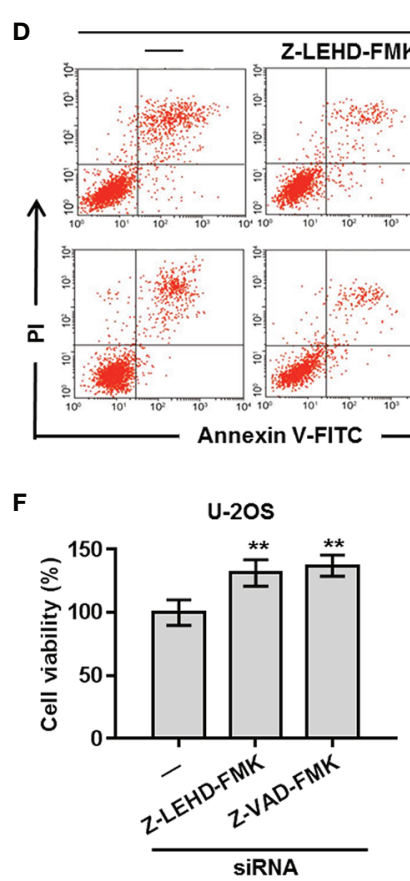

SiRNA

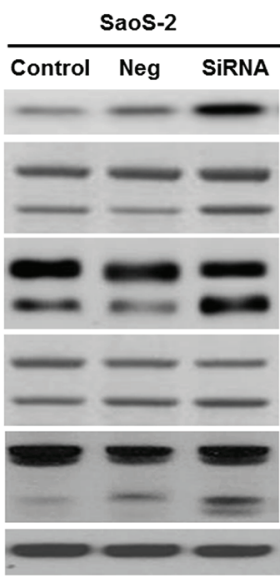

E

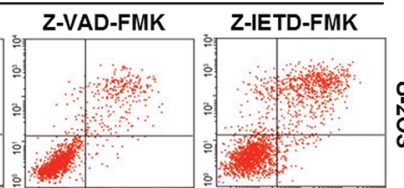

\section{灾}

$\square$ SiRNA Z-LEHD-FMK SIRNA Z-VAD-FMK $\overbrace{30}^{40}]$ T
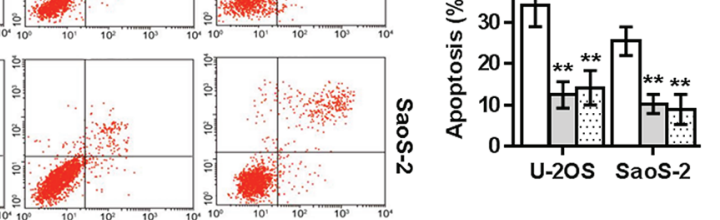

siRNA

SIRNA Z-IETD-FMK

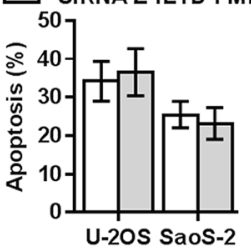

U-20s Saos-2

G

U-20s
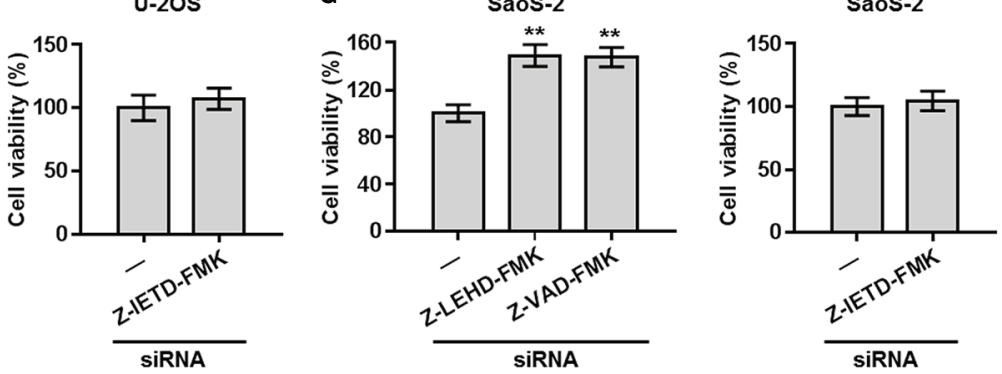

FIGURE 4 | Mitochondria-dependent apoptotic pathway is involved in the effect of ClC-5 knockdown on osteosarcoma cells. (A) The cytosol cytochrome c level, the active forms of caspase-9/8/3, and the cleavage product of PARP were determined in U-2OS and SaoS-2 cells treated with CIC-5 siRNA or negative siRNA (Neg). (B, C) Densitometric analysis of apoptosis-related protein expression was performed in U-2OS (B) and SaoS-2 (C) cells. ${ }^{* \star} \mathrm{P}<0.01$ vs. corresponding control, $\mathrm{n}=6$. (D, E) U-2OS and SaoS-2 cells were pretreated with caspase-9 inhibitor Z-LEHD-FMK (10 $\mu \mathrm{M})$, caspase-3 inhibitor Z-IETD-FMK (20 $\mu$ M), or caspase-8 inhibitor Z-VAD-FMK $(25 \mu \mathrm{M})$ for 30 min before CIC-5 siRNA transfection. Cell apoptosis was determined by Annexin V/PI staining (D). Quantitative analysis of the percentage of apoptotic cells (E). ${ }^{\star *} \mathrm{P}<0.01$ vs. siRNA, $n=4$. (F, G) Cell viability of U-2OS (F) and SaoS-2 (G) was determined. ${ }^{\star \star} P<0.01$ vs. siRNA, $n=6$.

the Bax content in cytosol and increased the content in mitochondria in U-2OS cells, indicating the ClC-5 knockdown induces Bax translocation from cytosol to mitochondria. The immunoprecipitation assay in U-2OS cells showed that ClC-5 was interacted with Bax (Figure 5C). It has been reported that tBid directly binds with Bax and promotes Bax translocation, leading to apoptosis initiation $(16,17)$. We thus investigated whether $\mathrm{ClC}-5$ influences the association between $\mathrm{tBid}$ and Bax. The results showed that $\mathrm{ClC}-5$ downregulation significantly increased the binding of Bax to tBid (Figure 5D). Collectively, the results suggest that $\mathrm{ClC}-5$ knockdown augments the interaction between Bax and $\mathrm{tBid}$, resulting to Bax translocation and apoptosis in osteosarcoma cells.

\section{DISCUSSION}

In this study, we found overexpression of ClC-5 in osteosarcoma tissues compared to normal bone tissues. Furthermore, our results demonstrated for the first time that $\mathrm{ClC}-5$-mediated $\mathrm{Cl}^{-}$ 
A

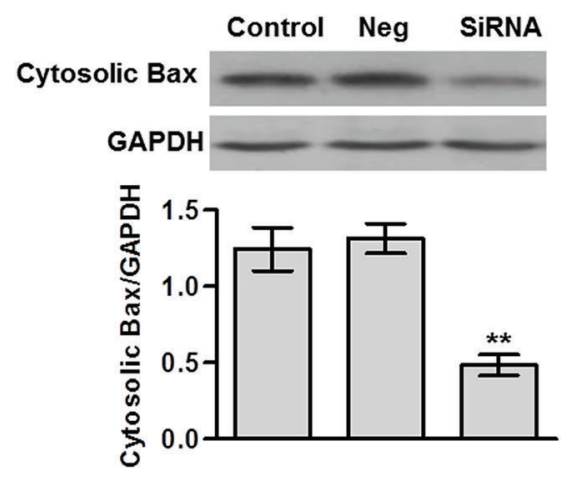

C

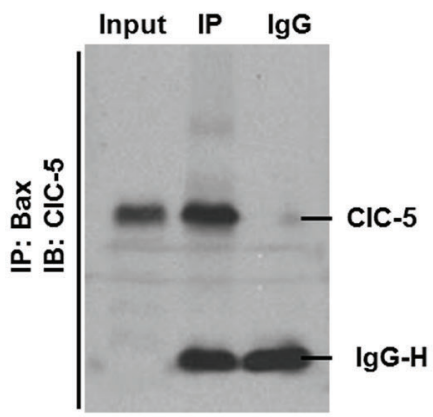

B

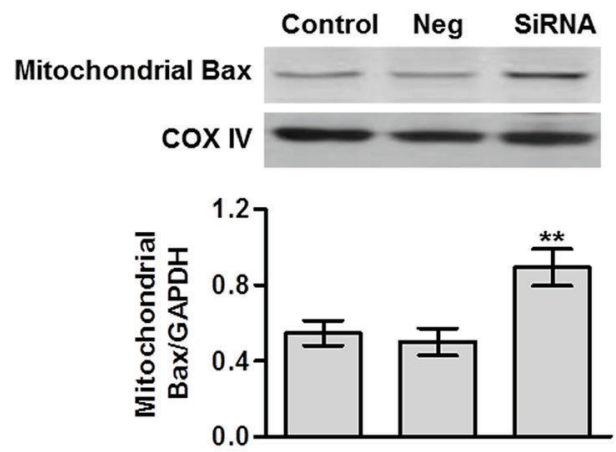

D
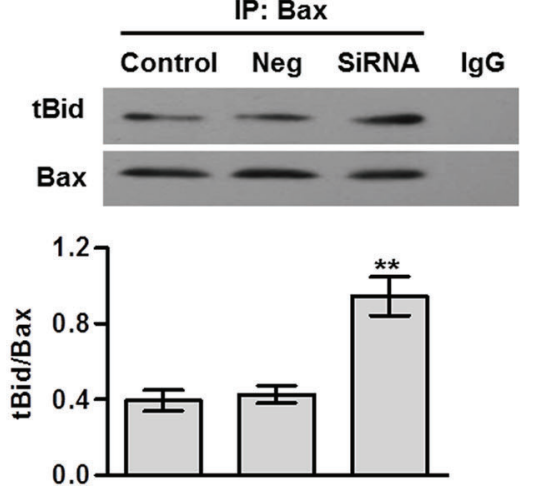

FIGURE 5 | ClC-5 interacts Bax and induces its translocation. (A and B) U-2OS osteosarcoma cells were transfected with CIC-5 siRNA or negative siRNA (Neg) for $48 \mathrm{~h}$. The Bax expression in cytosol (A) and mitochondria (B) fractions was determined by western blotting. ${ }^{\star \star} \mathrm{P}<0.01 \mathrm{vs}$. control, $\mathrm{n}=6$. (C) Cell lysates were immunoprecipitated (IP) with Bax antibody and the immunoprecipitated proteins were blotted with CIC-5 antibody. (D) Cell lysates from each group were immunoprecipitated with Bax antibody and blotted with tBid antibody. ${ }^{\star *} \mathrm{P}<0.01$ vs. control, $n=4$.

efflux is critical for the growth of osteosarcoma cells. Downregulation of $\mathrm{ClC}-5$ induced mitochondria-dependent apoptosis in osteosarcoma cells. These data suggest that ClC-5 may play a critical role in future osteosarcoma treatment strategy.

Increasing evidence has shown the importance of $\mathrm{Cl}^{-}$ channels in regulating the development of different tumors. For example, ClC-3 was found to be upregulated in glioma, breast, and cervical tumors (18-20). Overexpression of ClC-3 promoted the migration and invasion of nasopharyngeal and glioma cancer cells $(21,22)$. Inhibition of LRRC8A could effectively attenuate cancer drug resistance in glioma and ovarian cancer cells $(6,23)$. Additionally, knockdown of CFTR increased the sensitivity of prostate cancer cells to cisplatin treatment (24). However, the functions of ClC-5 in cancer cells are poorly understood. ClC- 5 has been found to be expressed in glioma cells and different leukemic cell lines $(11,12)$. ClC-5 overexpression inhibited bortezomib-induced the death of multiple myeloma cells (25). In this study, we are the first to demonstrate that $\mathrm{ClC}-5$ expression was upregulated in osteosarcoma tissues. Consistent with these results, ClC-5 expression was also increased in osteosarcoma cell lines compared with normal osteoblasts. The enrichment of ClC-5 in osteosarcoma cells was even close to that in renal proximal tubule epithelial HK-2 cells, where ClC-5 has been found to be highly expressed (13). Importantly, patients with ClC-5-high expression showed poorer survival relative to ClC-5-low patients, suggesting that the increased ClC- 5 expression may predict worst survival and serve as a critical prognostic indicator for osteosarcoma survival. Thus, these results indicate a key role of ClC-5 in osteosarcoma.

Interestingly, we found that the $\left[\mathrm{Cl}^{-}\right]_{\mathrm{i}}$ was decreased in osteosarcoma cells. Disturbance in $\mathrm{Cl}^{-}$homeostasis is widely implicated in the regulation of cell proliferation and apoptosis, including cancer cells (6-8). Yang et al. observed decreased $\mathrm{Cl}^{-}$efflux in temozolomide-resistant glioma cells, which was associated with inhibited cell apoptosis induced by temozolomide (6). Thus, in this study, we also explored whether the alteration in $\left[\mathrm{Cl}^{-}\right]_{\mathrm{i}}$ was involved in regulating the function of osteosarcoma cells. The results demonstrated that lowering $\left[\mathrm{Cl}^{-}\right]_{\mathrm{i}}$ using low $\mathrm{Cl}^{-}$culture medium increased the viability of osteosarcoma cells. However, the increased cell viability was inhibited by $\mathrm{ClC}-5$ downregulation. This strongly indicates that $\mathrm{ClC}-5$-mediated $\mathrm{Cl}^{-}$efflux promotes the growth of osteosarcoma cells. Subsequently, downregulation of ClC-5 markedly induced osteosarcoma cell apoptosis. It should be noted that lowering $\left[\mathrm{Cl}^{-}\right]_{\mathrm{i}}$ or downregulation of ClC-5 produced no significant effects on control hFOB1.19 cell 
viability, which may be associated with low abundance of ClC-5 in normal osteoblasts cells. These results further emphasize the importance of ClC-5 in the therapy of osteosarcoma.

It is well recognized that cell apoptosis is controlled by two major pathways: namely the extrinsic death receptor-mediate pathway and intrinsic then activated caspase-9, caspase-3, and PARP $(14,15)$. The former is activated at the plasma membrane via binding to extracellular ligand, such as FasL and TRAIL, leading to the cleavage of caspase- $8(26,27)$. On the other hand, the activation of intrinsic pathway results in cytochrome $c$ release from mitochondria to cytosol, which in turn cleaves caspase-9, caspase-3, and PARP (14). Thus, each apoptotic pathway activates corresponding initiator caspase, caspase-8, or caspase9. In this study, we found that $\mathrm{ClC}-5$ inhibition increased the release of cytochrome $c$ followed by activation of caspase-9, caspase-3, and PARP. However, ClC-5 knockdown had no effects on caspase- 8 activation, suggesting that ClC-5 downregulation activates the intrinsic pathway rather than extrinsic pathway. This was further supported by the subsequent results that caspase- 9 inhibitor or caspase-3 inhibitor reversed the proapoptotic effects of $\mathrm{ClC}-5$ downregulation.

We next investigated the mechanisms how ClC-5 knockdown causes cytochrome $\mathrm{c}$ release and initiates apoptosis. It is worthy to note that the release of cytochrome $c$ is triggered by the activation of Bax (16). Under normal conditions, Bax is mainly expressed in cytosol. Upon pro-apoptotic stimulation, Bax translates from cytosol to mitochondria and increases the membrane permeability, leading to cytochrome $c$ release and apoptosis initiation $(28,29)$. We found that ClC-5 downregulation induced Bax translocation from cytosol to mitochondria. More importantly, we reported for the first time that $\mathrm{ClC}-5$ is a novel Bax-interacting protein. Given that $\mathrm{BH} 3-$ only protein, such as tBid, can bind with Bax and help the translocation of Bax from cytosol to mitochondria $(17,30)$, the effects of ClC-5 knockdown on the interaction between Bax and tBid. We observed that $\mathrm{ClC}-5$ downregulation increased the binding of Bax to tBid, suggesting that $\mathrm{ClC}-5$ and tBid may competitively bind with Bax.

In conclusion, our results demonstrate that ClC-5 downregulation induces osteosarcoma cell apoptosis, which involves promotion of Bax and tBid complex formation, leading to cytochrome $\mathrm{c}$ release and intrinsic mitochondria-

\section{REFERENCES}

1. Ward E, DeSantis C, Robbins A, Kohler B, Jemal A. Childhood and adolescent cancer statistics, 2014. CA: Cancer J Clin (2014) 64(2):83-103. doi: 10.3322/ caac. 21219

2. Savage SA, Mirabello L, Wang Z, Gastier-Foster JM, Gorlick R, Khanna C, et al. Genome-wide association study identifies two susceptibility loci for osteosarcoma. Nat Genet (2013) 45(7):799-803. doi: 10.1038/ng.2645

3. Sakamoto A, Iwamoto Y. Current status and perspectives regarding the treatment of osteo-sarcoma: chemotherapy. Rev Recent Clin Trials (2008) 3 (3):228-31. doi: 10.2174/157488708785700267

4. Bielack SS, Marina N, Ferrari S, Helman LJ, Smeland S, Whelan JS, et al. Osteosarcoma: the same old drugs or more? J Clin Oncol Off J Am Soc Clin Oncol (2008) 26(18):3102-3; author reply 4-5. doi: 10.1200/JCO.2008.17.1108 dependent apoptotic pathway activation. These findings suggest that $\mathrm{ClC}-5$ may be a promising target for the treatment of osteosarcoma.

\section{DATA AVAILABILITY STATEMENT}

The raw data supporting the conclusions of this article will be made available by the authors, without undue reservation.

\section{ETHICS STATEMENT}

The studies involving human participants were reviewed and approved by Renmin Hospital of Wuhan University. The patients/participants provided their written informed consent to participate in this study. Written informed consent was obtained from the individual(s) for the publication of any potentially identifiable images or data included in this article.

\section{AUTHOR CONTRIBUTIONS}

Conception and design: FP. Administrative support: FP. Provision of study materials or patients: WC and JL. Collection and assembly of data: FP and HL. Data analysis and interpretation: FP. Manuscript writing: all authors. All authors contributed to the article and approved the submitted version.

\section{FUNDING}

This work was supported by the National Natural Science Foundation of China (631308110).

\section{SUPPLEMENTARY MATERIAL}

The Supplementary Material for this article can be found online at: https:/www.frontiersin.org/articles/10.3389/fonc.2020.556908/ full\#supplementary-material

5. Thompson EG, Sontheimer H. A role for ion channels in perivascular glioma invasion. Eur Biophys J EBJ (2016) 45(7):635-48. doi: 10.1007/s00249-0161154-X

6. Yang C, He L, Chen G, Ning Z, Xia Z. LRRC8A potentiates temozolomide sensitivity in glioma cells via activating mitochondria-dependent apoptotic pathway. Hum Cell (2018) 32(1):41-50. doi: 10.1007/s13577-018-0221-2

7. Yang H, Huang LY, Zeng DY, Huang EW, Liang SJ, Tang YB, et al. Decrease of intracellular chloride concentration promotes endothelial cell inflammation by activating nuclear factor-kappaB pathway. Hypertension (2012) 60(5):1287-93. doi: 10.1161/HYPERTENSIONAHA.112.198648

8. Wu QQ, Liu XY, Xiong LX, Shang JY, Mai XY, Pang RP, et al. Reduction of Intracellular Chloride Concentration Promotes Foam Cell Formation. Circ J Off J Jpn Circ Soc (2016) 80(4):1024-33. doi: 10.1253/circj.CJ-15-1209

9. Zhang H, Pang Y, Ma C, Li J, Wang H, Shao Z. ClC5 Decreases the Sensitivity of Multiple Myeloma Cells to Bortezomib via Promoting Prosurvival 
Autophagy. Oncol Res (2018) 26(3):421-9. doi: 10.3727/096504017 X15049221237147

10. Jiang B, Hattori N, Liu B, Nakayama Y, Kitagawa K, Sumita K, et al. Expression and roles of $\mathrm{Cl}-$ channel $\mathrm{ClC}-5$ in cell cycles of myeloid cells. Biochem Biophys Res Commun (2004) 317(1):192-7. doi: 10.1016/ j.bbrc.2004.03.036

11. Ernest NJ, Weaver AK, Van Duyn LB, Sontheimer HW. Relative contribution of chloride channels and transporters to regulatory volume decrease in human glioma cells. Am J Physiol Cell Physiol (2005) 288(6):C1451-60. doi: 10.1152/ ajpcell.00503.2004

12. Olsen ML, Schade S, Lyons SA, Amaral MD, Sontheimer H. Expression of voltage-gated chloride channels in human glioma cells. J Neurosci Off J Soc Neurosci (2003) 23(13):5572-82. doi: 10.1523/JNEUROSCI.23-13-05572.2003

13. Wang Y, Cai H, Cebotaru L, Hryciw DH, Weinman EJ, Donowitz M, et al. ClC-5: role in endocytosis in the proximal tubule. Am J Physiol Renal Physiol (2005) 289(4):F850-62. doi: 10.1152/ajprenal.00011.2005

14. Yang H, Liao D, Tong L, Zhong L, Wu K. MiR-373 exacerbates renal injury and fibrosis via NF-kappaB/MatrixMetalloproteinase- 9 signaling by targeting Sirtuin1. Genomics (2018) 111(4):786-92. doi: 10.1016/j.ygeno.2018.04.017

15. Li Z, Hong Z, Peng Z, Zhao Y, Shao R. Acetylshikonin from Zicao ameliorates renal dysfunction and fibrosis in diabetic mice by inhibiting TGF-beta1/Smad pathway. Hum Cell (2018) 31(3):199-209. doi: 10.1007/s13577-017-0192-8

16. Huang XZ, Wen D, Zhang M, Xie Q, Ma L, Guan Y, et al. Sirtl activation ameliorates renal fibrosis by inhibiting the TGF-beta/Smad3 pathway. J Cell Biochem (2014) 115(5):996-1005. doi: 10.1002/jcb.24748

17. Qiu Z, Dubin AE, Mathur J, Tu B, Reddy K, Miraglia LJ, et al. SWELL1, a plasma membrane protein, is an essential component of volume-regulated anion channel. Cell (2014) 157(2):447-58. doi: 10.1016/j.cell.2014.03.024

18. Xu B, Jin X, Min L, Li Q, Deng L, Wu H, et al. Chloride channel-3 promotes tumor metastasis by regulating membrane ruffling and is associated with poor survival. Oncotarget (2015) 6(4):2434-50. doi: 10.18632/oncotarget.2966

19. Mao J, Chen L, Xu B, Wang L, Wang W, Li M, et al. Volume-activated chloride channels contribute to cell-cycle-dependent regulation of HeLa cell migration. Biochem Pharmacol (2009) 77(2):159-68. doi: 10.1016/ j.bcp.2008.10.009

20. Becker GJ, Hewitson TD. The role of tubulointerstitial injury in chronic renal failure. Curr Opin Nephrol Hypertens (2000) 9(2):133-8. doi: 10.1097/ 00041552-200003000-00006

21. Waasdorp M, de Rooij DM, Florquin S, Duitman J, Spek CA. Proteaseactivated receptor-1 contributes to renal injury and interstitial fibrosis during chronic obstructive nephropathy. J Cell Mol Med (2018) 23(2):1268-79. doi: $10.1111 / \mathrm{jcmm} .14028$
22. Qi R, Yang C. Renal tubular epithelial cells: the neglected mediator of tubulointerstitial fibrosis after injury. Cell Death Dis (2018) 9(11):1126. doi: 10.1038/s41419-018-1157-x

23. Meng XM, Wang S, Huang XR, Yang C, Xiao J, Zhang Y, et al. Inflammatory macrophages can transdifferentiate into myofibroblasts during renal fibrosis. Cell Death Dis (2016) 7(12):e2495. doi: 10.1038/cddis.2016.402

24. Zhu Q, Li H, Liu Y, Jiang L. Knockdown of CFTR enhances sensitivity of prostate cancer cells to cisplatin via inhibition of autophagy. Neoplasma (2017) 64(5):709-17. doi: 10.4149/neo_2017_508

25. Buchtler S, Grill A, Hofmarksrichter S, Stockert P, Schiechl-Brachner G, Rodriguez Gomez M, et al. Cellular Origin and Functional Relevance of Collagen I Production in the Kidney. J Am Soc Nephrol JASN (2018) 29 (7):1859-73. doi: 10.1681/ASN.2018020138

26. Gao R, Chen J, Hu Y, Li Z, Wang S, Shetty S, et al. Sirt1 deletion leads to enhanced inflammation and aggravates endotoxin-induced acute kidney injury. PloS One (2014) 9(6):e98909. doi: 10.1371/journal.pone.0098909

27. Voss FK, Ullrich F, Munch J, Lazarow K, Lutter D, Mah N, et al. Identification of LRRC8 heteromers as an essential component of the volume-regulated anion channel VRAC. Science (2014) 344(6184):634-8. doi: 10.1126/ science. 1252826

28. Carraro-Lacroix LR, Lessa LM, Bezerra CN, Pessoa TD, Souza-Menezes J, Morales MM, et al. Role of CFTR and ClC-5 in modulating vacuolar $\mathrm{H}$ +-ATPase activity in kidney proximal tubule. Cell Physiol Biochem Int J Exp Cell Physiol Biochem Pharmacol (2010) 26(4-5):563-76. doi: 10.1159/ 000322324

29. Rajagopal M, Wallace DP. Chloride secretion by renal collecting ducts. Curr Opin Nephrol Hypertens (2015) 24(5):444-9. doi: 10.1097/MNH. 0000000000000148

30. Laverty G, Anttila A, Carty J, Reddy V, Yum J, Arnason SS. CFTR mediated chloride secretion in the avian renal proximal tubule. Comp Biochem Physiol Part A Mol Integr Physiol (2012) 161(1):53-60. doi: 10.1016/j.cbpa.2011.09.005

Conflict of Interest: The authors declare that the research was conducted in the absence of any commercial or financial relationships that could be construed as a potential conflict of interest.

Copyright $\odot 2021$ Peng, Cai, Li and Li. This is an open-access article distributed under the terms of the Creative Commons Attribution License (CC BY). The use, distribution or reproduction in other forums is permitted, provided the original author $(s)$ and the copyright owner(s) are credited and that the original publication in this journal is cited, in accordance with accepted academic practice. No use, distribution or reproduction is permitted which does not comply with these terms. 